\title{
Loss-of-function variants in myocardin cause congenital megabladder in humans and mice
}

\author{
Arjan C. Houweling, ${ }^{1}$ Glenda M. Beaman, ${ }^{2,3}$ Alex V. Postma, ${ }^{1,4}$ T. Blair Gainous, ${ }^{5}$ Klaske D. Lichtenbelt, ${ }^{6}$ Francesco Brancati, ${ }^{7,8}$ \\ Filipa M. Lopes, ${ }^{2,3}$ Ingeborg van der Made, ${ }^{9}$ Abeltje M. Polstra, ${ }^{1}$ Michael L. Robinson, ${ }^{10}$ Kevin D. Wright, ${ }^{10}$ Jamie M. Ellingford, ${ }^{2,3}$ \\ Ashley R. Jackson, ${ }^{11}$ Eline Overwater, ${ }^{1}$ Rita Cenesio, ${ }^{12}$ Silvio Romano, ${ }^{8}$ Letizia Camerota, ${ }^{8}$ Emanuela D'Angelo, ${ }^{8}$ \\ Elizabeth J. Meijers-Heijboer, ${ }^{1}$ Vincent M. Christoffels, ${ }^{4}$ Kirk M. McHugh, ${ }^{11}$ Brian L. Black, ${ }^{5}$ William G. Newman, ${ }^{2,3}$ \\ Adrian S. Woolf, ${ }^{2,3}$ and Esther E. Creemers ${ }^{9}$ \\ 'Department of Clinical Cenetics, Amsterdam UMC, Amsterdam, Netherlands. ${ }^{2}$ School of Biological Sciences, Faculty of Biology, Medicine and Health, University of Manchester, Manchester, United Kingdom. \\ ${ }_{3}^{3}$ Manchester Centre for Genomic Medicine and Royal Manchester Children's Hospital, Manchester University NHS Foundation Trust, Manchester Academic Health Science Centre, Manchester, United \\ Kingdom. ${ }^{4}$ Department of Medical Biology, Amsterdam UMC, Amsterdam, Netherlands. ${ }^{5}$ Cardiovascular Research Institute, UCSF, San Francisco, California, USA. ${ }^{6}$ Department of Medical Genetics, University \\ Medical Center Utrecht, Utrecht, Netherlands. 'aboratory of Molecular and Cell Biology, Istituto Dermopatico dell'Immacolata, IDI-IRCCS, Rome, Italy. ${ }^{8}$ Department of Life, Health and Environmental \\ Sciences, University of L'Aquila, Aquila, Italy. ${ }^{9}$ Department of Experimental Cardiology, Amsterdam UMC, Amsterdam, Netherlands. ${ }^{10}$ Department of Biology, Miami University, Oxford, Ohio, USA. "'Center \\ for Clinical and Translational Research, The Research Institute, Nationwide Children's Hospital, Columbus, Ohio, USA. ${ }^{2}$ Department of Molecular Medicine and Medical Biotechnology, University of Naples \\ Federico II, Naples, Italy.
}

\begin{abstract}
Myocardin (MYOCD) is the founding member of a class of transcriptional coactivators that bind the serum-response factor to activate gene expression programs critical in smooth muscle (SM) and cardiac muscle development. Insights into the molecular functions of MYOCD have been obtained from cell culture studies, and to date, knowledge about in vivo roles of MYOCD comes exclusively from experimental animals. Here, we defined an often lethal congenital human disease associated with inheritance of pathogenic MYOCD variants. This disease manifested as a massively dilated urinary bladder, or megabladder, with disrupted SM in its wall. We provided evidence that monoallelic loss-of-function variants in MYOCD caused congenital megabladder in males only, whereas biallelic variants were associated with disease in both sexes, with a phenotype additionally involving the cardiovascular system. These results were supported by cosegregation of $M Y O C D$ variants with the phenotype in 4 unrelated families by in vitro transactivation studies in which pathogenic variants resulted in abrogated SM gene expression and by the finding of megabladder in 2 distinct mouse models with reduced Myocd activity. In conclusion, we have demonstrated that variants in MYOCD result in human disease, and the collective findings highlight a vital role for MYOCD in mammalian organogenesis.
\end{abstract}

\section{Introduction}

Urinary tract and kidney malformations often result in termination of pregnancy after being detected on ultrasound screening, and these anomalies are also a major cause of renal failure in surviving children (1). While the genetic bases of kidney malformations are well-recognized (2), with pathogenic variants reported in transcription and growth factors that drive metanephric differentiation, the possible genetic causes of congenital ureter and bladder anomalies are much less clear (3). A striking urinary tract phenotype is megabladder, with a first trimester prevalence of 1:330-1670 (4). Megabladder accompanied by a thinned and wrinkled abdominal wall overlying the bladder is called prune

Authorship note: $A C H, G M B$, and AVP contributed equally to this work. WCN, ASW, and EEC jointly supervised this work.

Conflict of interest: The authors have declared that no conflict of interest exists. Copyright: () 2019, Houweling et al. This is an open access article published under the terms of the Creative Commons Attribution 4.0 International License.

Submitted: March 1, 2019; Accepted: September 3, 2019; Published: November 4, 2019. Reference information: J Clin Invest. 2019;129(12):5374-5380.

https://doi.org/10.1172/JCl128545. belly syndrome (PBS). Megabladder and PBS are accompanied by kidney glomerular cysts considered to be secondary to fetal urinary flow obstruction (5). Some megabladders are associated with anatomically obstructed bladder outflow tracts, and these bladders have increased smooth muscle (SM) in their walls. Other megabladders are examples of functional outflow obstruction and have patent urethras and thin bladder walls (6). We hypothesized that mutations in genes affecting SM differentiation can cause megabladder and PBS.

Myocardin (MYOCD) is the founding member of a class of powerful transcriptional coactivators that bind to serum response factor (SRF) to activate cardiac- and SM-specific gene expression programs $(7,8)$. Complete loss of Myocd in mice causes embryonic lethality at midgestation due to failure of SM cell differentiation, whereas heterozygous knockout mice appear normal (9). Conditional mouse models subsequently defined the function of Myocd in postnatal development. Specifically, genetic ablation of Myocd in adult hearts causes heart failure due to loss of sarcomere structure and increased cardiomyocyte apoptosis (10). Genetic deletion of Myocd specifically in SM revealed that Myocd maintains vascular and visceral SM homeostasis postnatally (11). Despite these 
A
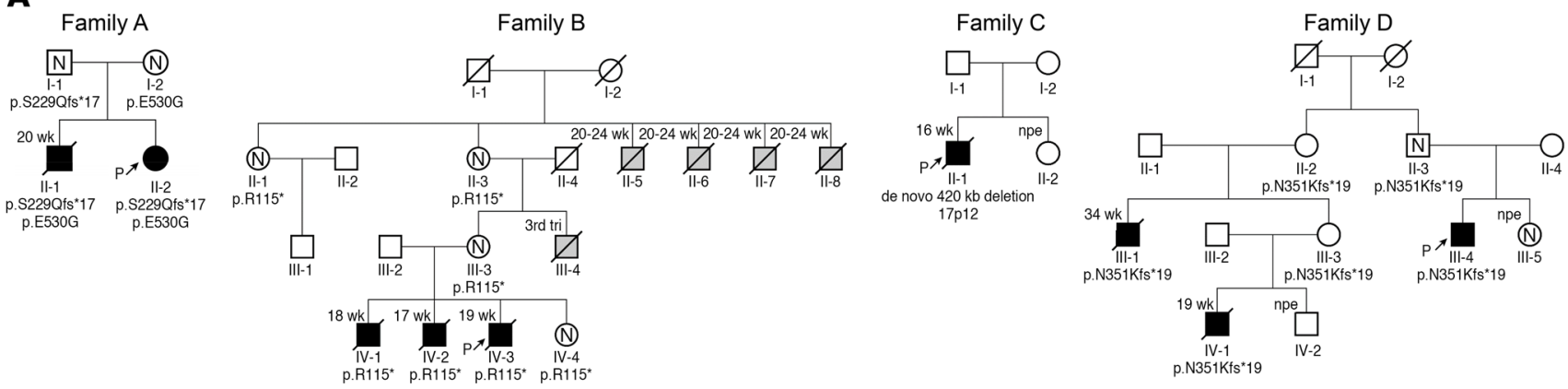

\section{B}

Human

Chimpanzee

Rhesus

Wolf

Cow

Mouse

Rat

Chicken

Zebrafish

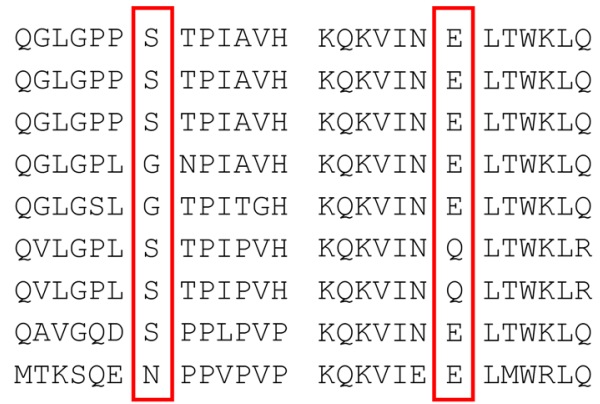

Family A mutation 1 p.S229Qfs*17 c.684dupC
Family A mutation 2

p.E530G

c. $1589 A>G$

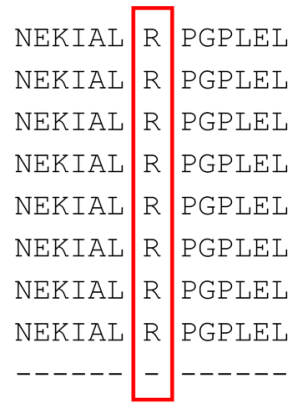

Family B

p.R115*

c. $343 \mathrm{C}>\mathrm{T}$

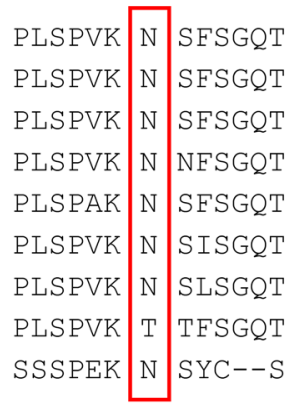

Family D

p.N351Kfs*19

c.1053-1054del

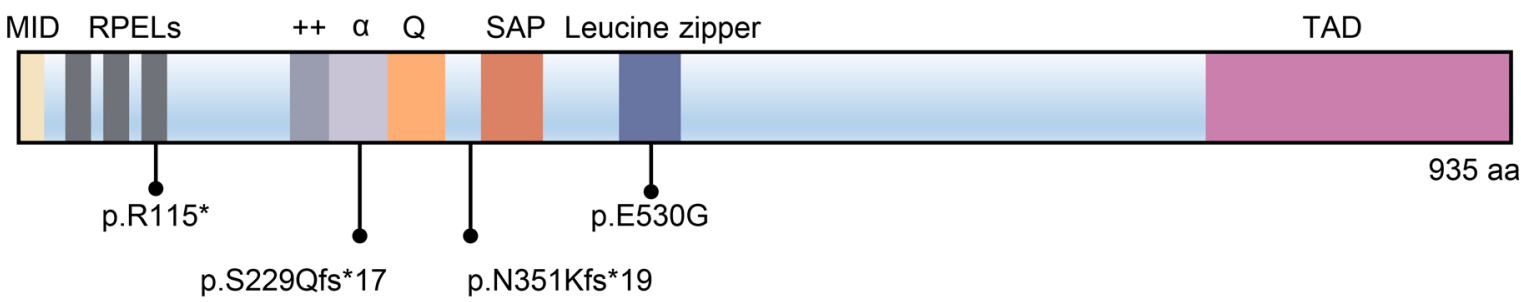

C
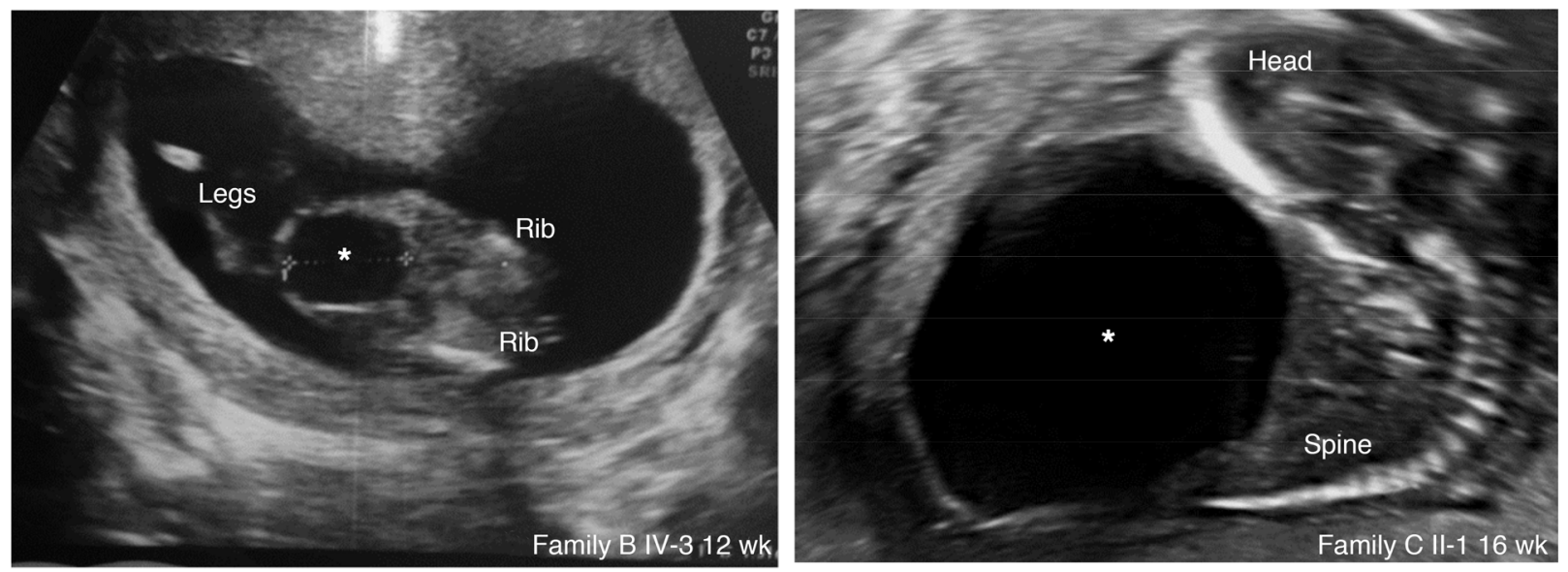

Figure 1. Identification of $M Y O C D$ variants in $\mathbf{4}$ families with congenital megabladder. (A) Pedigrees of 4 families presenting with congenital megabladder. Affected individuals are marked with black symbols. Available genotypes are shown beneath symbols. Slashed symbols denote deceased individuals. Gestational age is indicated above the symbol. Gray symbols denote stillbirths with external features consistent with PBS. N, normal bladder ultrasound; $P$ with arrow, proband of the family; npe, normal prenatal echo. (B) Schematic diagram showing functional domains within MYOCD and location of the identified mutations (7). Conservation of respective amino acid positions with the mutated residues are highlighted. (C) Ultrasound images showing enlarged bladder of indicated fetuses of families B and C; asterisks denote bladder. 

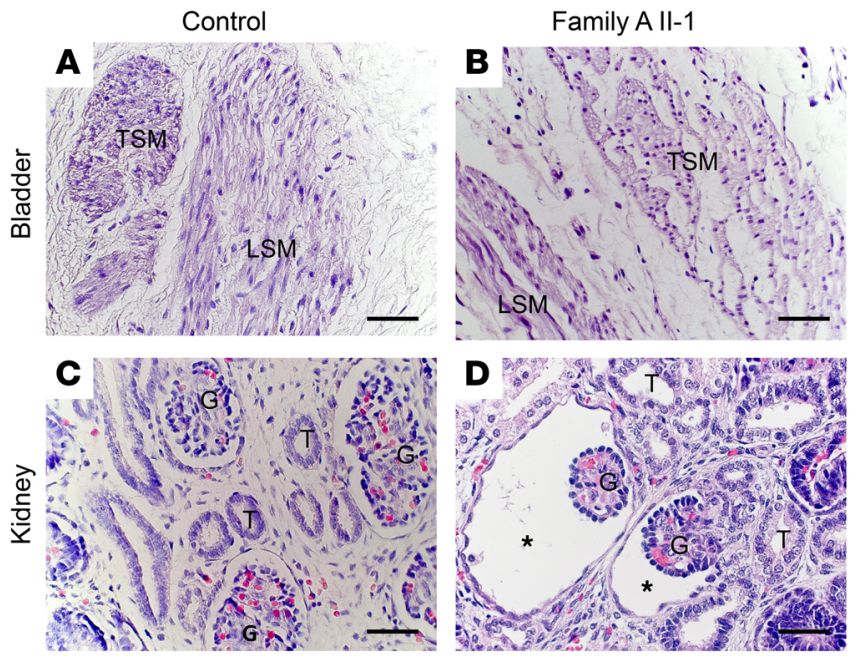

Figure 2. Bladder and kidney abnormalities in family $\mathbf{A}$. (A and C) From healthy midgestation fetuses. (B and $\mathbf{D}$ ) From affected fetus from family A. H\&E staining from urinary bladders shows transverse sections of muscle bundles (TSM) and longitudinal sections of muscle bundles (LSM) in the healthy and affected fetuses. Note, however, that the bundles in the affected fetus appear disorganized and less compact compared with the well-defined muscle fibers in the control. (C) In a control fetal kidney, glomeruli (C) and tubules (T) are evident. (D) In the kidney from the affected fetus, glomeruli are cystic, with dilated Bowman's spaces (asterisks), a characteristic of fetal urinary flow obstruction. Scale bars: $20 \mu \mathrm{m}$.

unique and important functions of Myocd in mice, no human genetic disorder associated with $M Y O C D$ variants has yet been defined. Here, we describe $M Y O C D$ loss-of-function variants in 9 individuals from 4 families with the megabladder/PBS spectrum, and we support these observations on urinary tract maldevelopment with Myocd mutant mouse models.

\section{Results and Discussion}

The index patient of family A (II-2) (Figure 1A) had a history of antenatal megabladder, and a bladder diverticulum was surgically resected in infancy. At 32 years of age, cardiac evaluation showed noncompaction cardiomyopathy and marked dilation $(51 \mathrm{~mm})$ of the aortic root. She also had an atrial septal defect, a ventric- ular septal defect (VSD), a patent ductus arteriosus, and a bicuspid aortic valve. Her brother (II-1) had been diagnosed prenatally with megabladder and VSD, prompting clinical termination of pregnancy at midgestation. Autopsy revealed PBS, and histology showed disorganized SM bundles in the bladder and glomerular cysts in the kidneys (Figure 2). The distal urethra was patent (Supplemental Figure 1; supplemental material available online with this article; https://doi.org/10.1172/JCI128545DS1), but the prostate and proximal urethra were not identified. A conclusion regarding anatomical obstruction was not possible because formal tests of urethral patency were not undertaken. The hindgut contained only a defined circular SM layer (Supplemental Figure 2) and lacked the longitudinal layer that should be present at this gestational age. In contrast, 2 normal SM layers were present in the small intestine and pulmonary artery SM appeared normal (Supplemental Figure 2). Ultrasonography revealed no bladder or heart abnormalities in the parents. Whole-exome sequencing (WES) in index patient II-2 and Sanger sequencing in II-1 determined that both siblings carried compound heterozygous variants in $M Y O C D$ (p.[S229Qfs*17];[E530G], respectively called family A mutation 1 and family A mutation 2 in Figure 1A). The variant p.[S229Qfs*17] is predicted to create a premature stop codon and was paternally inherited, while the missense variant p.[E530G] is located in the functional leucine zipper (LZ) of the encoded protein $(12,13)$ and was maternally inherited (Figure 1, A and B). Both variants were absent from over 120,000 control exomes (14). WES in the index case failed to reveal pathogenic variants in genes known to cause megabladder, including ACTG2, CHRM3, HPSE2, LRIG2, smooth muscle myosin heavy chain 11 (MYH11), and myosin light chain kinase (MYLK) $(3,15-17)$.

Next, we ascertained 22 additional families with megabladder or PBS of unknown etiology, identifying 7 affected individuals from 3 unrelated families, all with heterozygous predicted loss-offunction MYOCD variants (Figure 1A and Supplemental Table 1). In family B, there were 3 male fetal deaths, all with PBS (Figure $1, \mathrm{~A}$ and $\mathrm{C}$ ). WES revealed a heterozygous variant, c.343C $>\mathrm{T}$, in these 3 brothers, predicted to result in a premature stop p.[R115*]. This variant was also present in the unaffected mother, unaffected maternal grandmother, and a healthy female sibling, each with normal bladder and heart imaging. The grandmother (II-3)
A

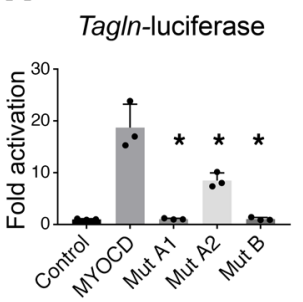

\section{B}

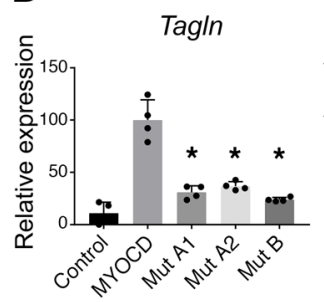

Myh11

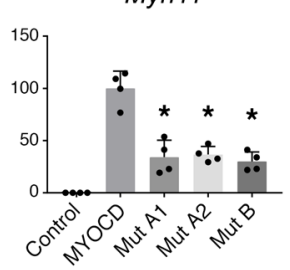

Cnn1

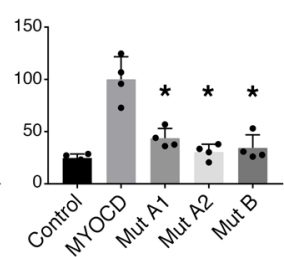

Mylk

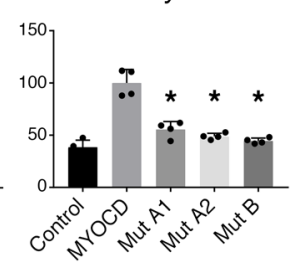

Acta2

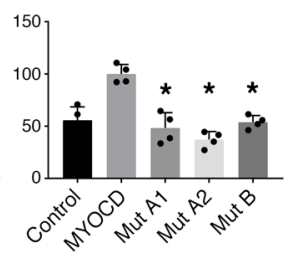

Figure 3. MYOCD mutations abrogate activation of SM cell gene expression in vitro. (A) Mouse fibroblasts were transiently transfected for 48 hours with expression vectors for MYOCD or the indicated MYOCD mutants (mutation A1: p.S229Qfs*17; mutation A2: p.E530G; mutation B: p.R115*) and a luciferase reporter linked to the Transgelin (Sm22) promoter ( $n=3 /$ group). (B) Mouse fibroblasts were transfected with expression plasmids encoding MYOCD or the indicated mutants ( $n=4$ /group). An empty expression plasmid served as a control. RNA was isolated, and SM gene expression was measured by qPCR. GAPDH was used to normalize expression. Overexpression levels of MYOCD were comparable between conditions (Supplemental Figure 3). ${ }^{*} P<0.01$ compared with WT MYOCD according to 1-way ANOVA with Dunnett's multiple comparison test. Shown are representative experiments of 2 independent repeats. 
A
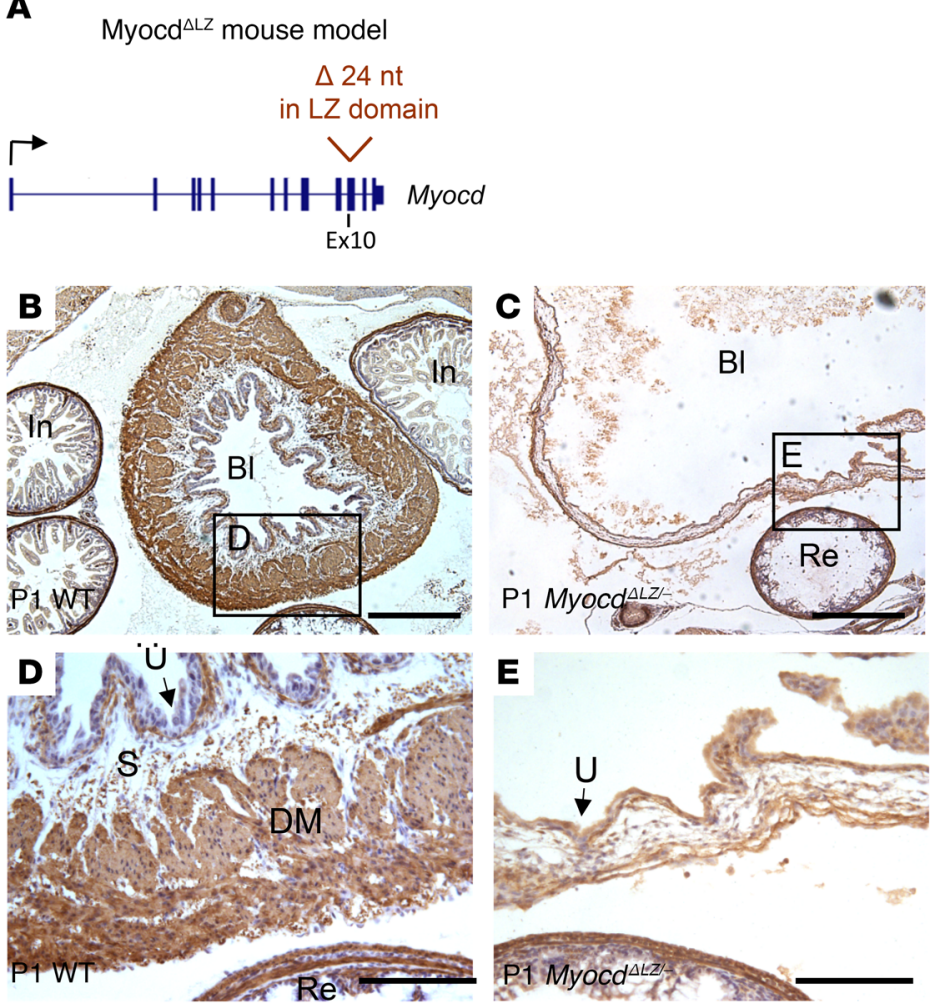

Figure 4. Myocd loss of function in mice causes the megabladder phenotype. (A) Schematic representation of the Myocd $^{\Delta L Z}$ allele, in which 24 nucleotides are deleted in the $L Z$ domain (p.I531_R539delinsM in NP_666498.2). (B-E) aSMA immunohistochemistry in 1-day-old neonates from $\mathrm{Myocd}^{+/-}$ and Myocd ${ }^{\Lambda L Z /+}$ crosses. (C) Compound heterozygosity (Myocd $^{\Delta L z /-}$; reminiscent of the alleles present in the affected individuals in family $A$ ) results in wall thinning of the bladder and lack of SM cells compared with the WT bladder wall (B). (D and E) Higher magnifications of WT and Myocd ${ }^{\triangle L Z /-}$ bladder walls showing lack of $\alpha \mathrm{SMA}$-expressing muscle bundles in the putative detrusor layer, although expression appeared retained in myofibroblast-like cells in the lamina propria directly below the urothelium and in the rectum. (F) Schematic representation of the Myocd ${ }^{\mathrm{Mgb}}$ allele. (G-J) Representative aSMA immunohistochemistry in P1 bladder of WT (G and $\mathbf{H})$ and Myocd ${ }^{m g b /-}$ compound heterozygote (I and J) (from a cross of $\mathrm{Myocd}^{\mathrm{mgb/+}}$ and $\mathrm{Myocd}^{+/-}$mice). Note the severe bladder distention and absent detrusor muscle in the Myocd ${ }^{\mathrm{mgb} /}$ bladder. BI, bladder; In, intestine; Re, rectum; U, urethelium; S, submucosa; DM, detrusor muscle. Scale bars: $500 \mu \mathrm{m}$ (B, C, G, and I); $100 \mu \mathrm{m}$ (D, E, H, and J). (K) Myocd mRNA levels were quantified by qPCR using E15 bladders of WT, Myocd+/, Myocd $^{m g b /+}$, Myocd $^{m g b / m g b}$, and Myocd ${ }^{m g b /-}$ mice. The absolute numbers of embryos developing megabladder as a fraction of the total number of embryos analyzed are indicated above the graph and reveals a highly penetrant phenotype in the Myocd $^{m g b / m g b}$ and Myocd ${ }^{m g b /-}$ mice.

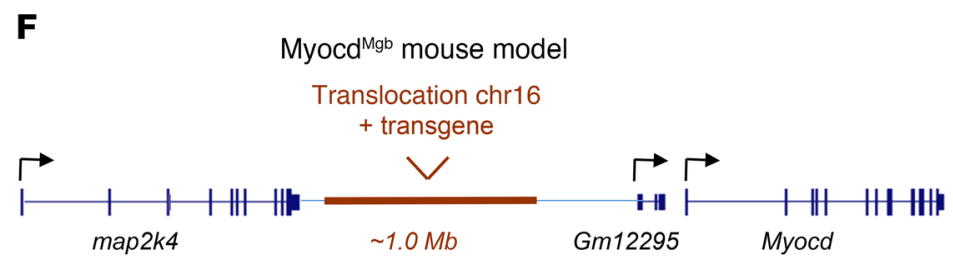

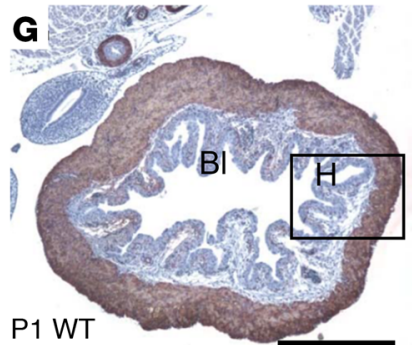
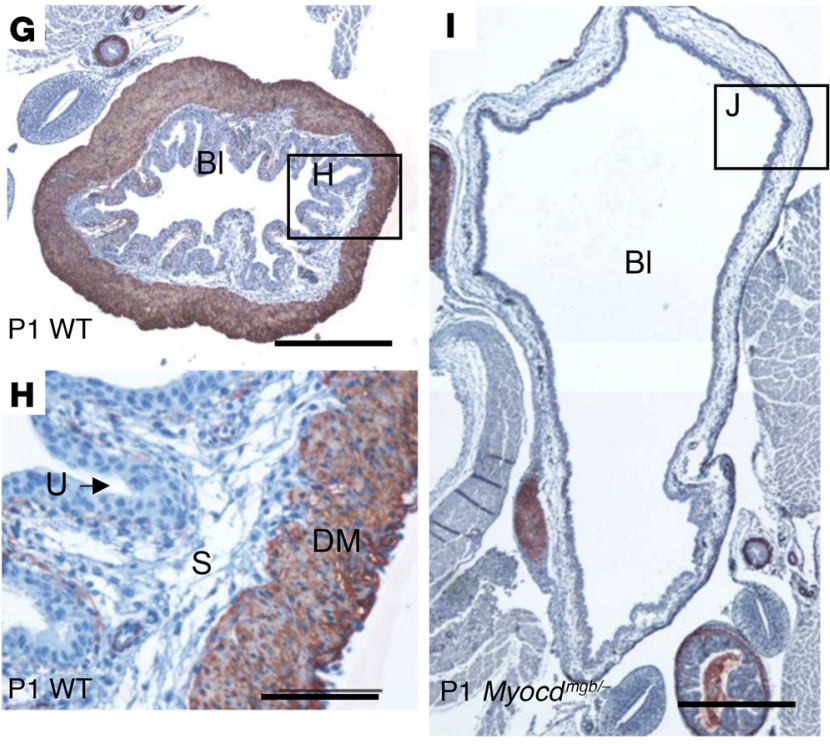

reported a male stillbirth (III-4) of unknown cause in the third trimester. She had 5 siblings, including 4 brothers who died antenatally (II-5, II-6, II-7, and II-8), each with a megabladder (further details unavailable), and 1 healthy sister (Figure 1A). Family
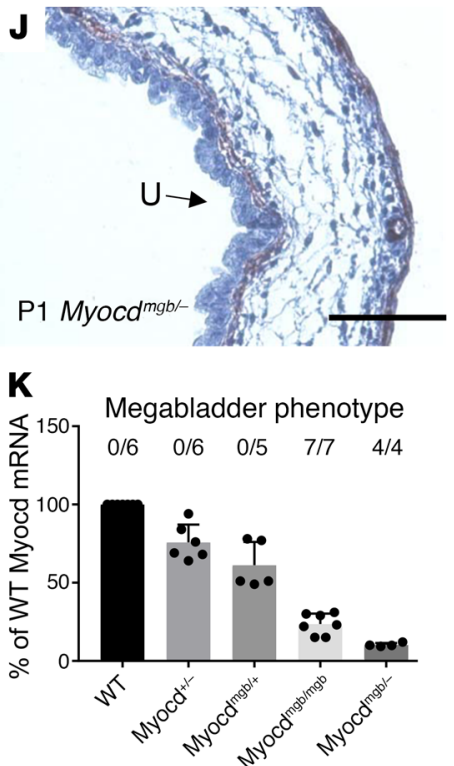

C's first pregnancy was terminated after diagnosis of PBS in the male fetus (Figure 1, A and C). Chromosomal microarray analysis revealed a heterozygous de novo $420 \mathrm{~kb}$ deletion (chr17p12; hg19:12,172,568-12,609,597) encompassing the first 2 exons of 
MYOCD, including the start codon. In family D, 3 males had PBS: 2 died prenatally (III-1 and IV-1), and the other (III-4) was born and underwent kidney transplantation for end-stage renal failure (Figure 1A). Sanger sequencing of MYOCD identified a heterozygous deletion of a single base c.1053-1054del, resulting in a predicted frameshift $\mathrm{p}$.[N351Kfs $\left.{ }^{*} 19\right]$.

We tested the abilities of proteins encoded by MYOCD variants from families A and B to activate the promoter of transgelin (Sm22 or transgelin [Tagln]), encoding a SM contractile protein. Western blotting revealed that the 2 predicted nonsense variants (p.[S229Qfs $\left.{ }^{\star} 17\right]$ mutation A1 from family A and p.[R115*] mutation $\mathrm{B}$ from family B) produced a truncated protein, whereas the missense variant (E530G, mutation A2 from family A) produced full-length protein (Supplemental Figure 3 and Supplemental Table 3). Neither nonsense variant resulted in activation of the Tagln-luciferase reporter, whereas the missense variant resulted in diminished activity versus WT MYOCD (Figure 3A). The above results regarding the missense variant are consistent with previous reports showing that $M Y O C D$ homodimerizes through the LZ domain and that homodimerization facilitates stronger activation of SRF-dependent reporter genes $(8,12)$. Myocd is normally not expressed in 10T1/2 fibroblasts, but experimental overexpression in these cells activates the SM differentiation program (8). We transfected 10T1/2 cells with either WT MYOCD or one of each of the 3 variants from families A and B. As assessed by quantitative PCR (qPCR), WT MYOCD strongly induced endogenous expression of the SM transcripts Tagln, Myh11, Calponin 1 (Cnn1), Mylk, and SM actin alpha 2 (Acta2). Conversely, each of the 3 MYOCD variants resulted in either a lack of increased expression or statistically significantly blunted responses (Figure 3B).

To assess whether reduced MYOCD activity causes megabladder, we took advantage of a newly generated mutant mouse line carrying an allele, Myocd ${ }^{\Delta L Z}$, in which critical residues within the LZ of Myocd were specifically deleted (Figure 4A and Supplemental Figure 4A). Using primers that distinguish the WT from the LZ mutated transcript, we showed that both were detected in neonatal bladders of Myocd $^{\Delta L Z /+}$ mice (Supplemental Figure 4B). We crossed Myocd ${ }^{\Delta L Z /+}$ mice with those carrying a null allele of Myocd $\left(\right.$ Myocd $\left.^{+-}\right)$(18). The alleles in the compound mutant offspring $\left(\right.$ Myocd $\left.^{4 L Z /-}\right)$ therefore mimicked the LZ and nonsense mutant alleles in family A. In contrast to homozygous null Myocd mutants (Myocd $\left.{ }^{-/-}\right)$(9), compound heterozygous mutant offspring ( Myocd $\left.^{\Delta L Z /-}\right)$ survived to birth. In line with the human urinary tract malformations, Myocd $^{4 L Z /-}$ mice developed grossly dilated bladders with little or no SM in their walls (Figure 4, A-E, and Supplemental Figure 4, C and D). In Myocd ${ }^{4 L Z /-}$ mouse bladders, transcript levels of several Myocd target genes (i.e., Acta2, Myh11, Mylk, Tagln, and Cnn1) were blunted versus those of WTs (Supplemental Figure 4E).

To gain further insight into the potential role of Myocd gene dosage in bladder malformations, we examined the megabladder mouse $(m g b)$ generated by random insertion and translocation of a transgene into chromosome 11 (19). Transcriptional profiling in the bladders of these mice had already revealed that levels of Myocd transcripts were significantly reduced (20). Here, we identified the translocation breakpoint (together with 4 copies of translocated chr. 16 region) approximately $500 \mathrm{~kb}$ upstream of the Myocd gene
(Figure 4F, Supplemental Figure 5, and Supplemental Table 4), suggesting the presence of a regulatory Myocd enhancer. Next, we crossed Myocd $^{m b b /+}$ with Myocd $^{+/-}$mice (9) and demonstrated that compound mutant offspring (Myocd $\left.{ }^{m g b /-}\right)$ had megabladders, providing further evidence that marked reduction of Myocd causes this phenotype (Figure 4, G-J, and Supplemental Figures 6 and 7). In addition, we observed patent ductus arteriosus in newborn Myocd $^{\text {mgb/- }}$ mice, but not in Myocd $^{+/-}$mice with just 1 allele mutated (Supplemental Figure 8). By studying various Myocd mutants (Figure $4 \mathrm{~K}$ ), we showed that a $70 \%$ to $80 \%$ reduction in Myocd mRNA in the bladder is sufficient to produce megabladder in mice. Interestingly, neonatal Myocd ${ }^{\mathrm{mgb} /-}$ mouse bladders have severely reduced transcript levels of Myocd target genes, yet in aortas and hearts of these same mice, the levels of Myocd target transcripts (apart from Myh11 in the aorta) are similar to those of heterozyous (Myocd $^{\mathrm{mb} /+}$ ) controls (Supplemental Figure 9).

The bladder phenotypes in families carrying MYOCD variants are thus mimicked by 2 distinct mouse models with reduced Myocd activity. Supplemental Table 2 gives an overview of the phenotypes observed in the mouse lines. The collective results demonstrate that MYOCD plays a unique role in proper development of the bladder wall. Reduced Myocd activity resulted in little or no SM differentiation in the mouse bladders and in disorganized SM bundles in the human fetal bladder. These SM defects would diminish the muscular force required to void urine from the bladder, resulting in the functional equivalent of lower urinary tract obstruction with severe bladder distension, ultimately culminating in kidney failure and death $(19,21)$.

Megabladder/PBS is a sex-limited trait with $95 \%$ male predominance, likely the result of differences in urethra and bladder development and length differences in urethra between males and females $(4,6)$. Additionally, sex hormones may play a role in defining the severity and progression of the disease, as clinical evidence demonstrates increased male susceptibility to acute and chronic kidney injury $(22,23)$. Indeed, in our study, 7 of 8 males proven to carry heterozygous loss-of-function MYOCD variants died before birth, whereas all 6 female carriers with heterozygous loss-offunction variants appeared healthy. Consistent with this observation, male $M y o c d^{m b b / m g b}$ mice were also more severely affected than females (Supplemental Table 2 and ref. 19). Notably, the only female with bladder disease in our study carried biallelic variants in $M Y O C D$, suggesting that further reduction in MYOCD levels is needed to cause bladder phenotypes in females. This is supported by both Myocd mouse models in which compound heterozygosity (i.e., Myocd ${ }^{m g b /-}$ or Myocd $^{L Z /-}$ ) caused megabladder in either sex (Supplemental Table 2). An intriguing feature is the incomplete penetrance of bladder disease in a healthy male MYOCD mutation carrier in family D. This may be caused by allelic imbalance, where the penetrance of a dominant loss-of-function mutation is determined by the expression level of the second allele, for instance, due to variants in promoter or enhancer regions of MYOCD. Alternatively, redundancy with other MYOCD family members, such as MRTF-A, which is also expressed in developing bladders (24), may affect penetrance.

Overall, we propose that we have identified a semidominant disorder (25) in which heterozygous loss-of-function variants in MYOCD cause congenital megabladder, while biallelic loss-of- 
function $M Y O C D$ variants also cause a cardiovascular phenotype. Both biallelic carriers developed congenital heart defects, while the affected female was found, when investigated as an adult, to have severe dilation of the aortic root. A similar association of cardiac defects was observed in the Myocd ${ }^{m g b /}$ - mice (ref. 26 and Supplemental Figure 8). Notably, a previous study already hinted at the possible involvement of MYOCD in megabladder, as it described one sporadic case with PBS and a $1.3 \mathrm{Mb}$ deletion of multiple genes, including MYOCD (27). Other SM-related genes have been implicated in PBS. These include variants in ACTA2, a MYOCD target gene, as well as MYH11 and MYLK, in which variants can cause visceral myopathy, a phenotype encompassing megabladder $(3,16)$. Moreover, each of these 3 genes has been associated with inherited thoracic aortic aneurysm and dissection (28). Notably, an SMrestricted deletion of Myocd in mice causes dilation of several visceral organs, including the bladder, as well as dilation of the aorta (11). Hence, there is compelling evidence that reduced MYOCD levels can result in urological and cardiovascular disease.

In conclusion, we demonstrate for what we believe is the first time that variants in $M Y O C D$ result in human disease. We propose that monoallelic loss-of-function variants in $M Y O C D$ cause congenital megabladder in males and that biallelic variants are associated with disease manifest in females that also involves the cardiovascular system. These findings not only have important implications for genetic counseling of families with megabladder, but also shed light on bladder development and expand the pathophysiological spectrum of inherited SM disorders.

\section{Methods}

Experimental procedures are provided in Supplemental Methods.

Study approval. Blood samples for genetic testing were obtained upon written consent. Informed consent for DNA studies, clinical records, and use of ultrasound pictures and histological analysis of the terminated fetus of family A was obtained. Control human embryonic material, collected with maternal consent and ethical approval (REC 08/ H0906/21+5 and REC 18/NE/0290), was sourced from the MRC and Wellcome Trust Human Developmental Biology Resource. Mice were maintained according to the NIH Guide for the Care and Use of Labora- tory Animals (National Academies Press, 2011). All experiments using Myocd $^{\Delta L Z}$ mice complied with federal and institutional guidelines and were reviewed and approved by the UCSF IACUC. The Mgb mouse studies were approved by the IACUC of the Nationwide Children's Hospital.

\section{Author contributions}

$\mathrm{ACH}, \mathrm{GMB}$, and AVP share first authorship, and the order in which they are listed has been determined by workload. ACH, GMB, AVP, WGN, ASW, and EEC designed the study. TBG, FML, IVDM, AMP, MLR, KDW, RG, LC, and ED performed experiments. ACH, EO, SR, KDL, and FB contributed clinical samples and clinical data. ACH, JME, GMB, AVP, and WGN performed genetic analysis. ACH, GMB, AVP, KMM, ARJ, BLB, WGN, ASW, and EEC analyzed experimental data. BLB, KMM, and MLR contributed mouse lines. EJMH and VMC were instrumental in interpretation of the data. ACH, AVP, ASW, and EEC wrote the manuscript.

\section{Acknowledgments}

We acknowledge support from the Medical Research Council (MR/L002744/1 to ASW and WGN); Horizon 2020 Marie Sklodowska-Curie RENALTRACT (942937 to ASW and FML); the Newlife Foundation (to ASW, GMB, and WGN); and the NIH (EY12995 to MLR; DK70907 and DK085242 to KMM; and HL064658, HL089707, and HL136182 to BLB). We also acknowledge an Out-of-the-Box Grant ACS (to ACH and EEC), a CVON CONCOR genes grant (to AVP and VMC), and support from Fondi di Ateneo, University of L'Aquila, and Undiagnosed Disease Network Italy at Istituto Superiore di Sanità (PGR00229-919 and Farmindustria to FB). We acknowledge Marilina Scalona and Fulvio De Simone for family B ascertainment.

Address correspondence to: Arjan C. Houweling, Departments of Clinical Genetics, Amsterdam UMC, De Boelelaan 1118, 1081HZ Amsterdam, Netherlands. Phone: 31204440150; Email: a.houweling@amsterdamumc.nl. Or to: Esther E. Creemers, Department of Experimental Cardiology, Amsterdam UMC, Meibergdreef 15, 1105AZ Amsterdam, Netherlands. Phone: 31205663262; Email: e.e.creemers@amsterdamumc.nl.
1. Wiesel A, Queisser-Luft A, Clementi M, Bianca S, Stoll C; EUROSCAN Study Group. Prenatal detection of congenital renal malformations by fetal ultrasonographic examination: an analysis of 709,030 births in 12 European countries. Eur JMed Genet. 2005;48(2):131-44.

2. Hildebrandt F. Genetic kidney diseases. Lancet. 2010;375(9722):1287-1295.

3. Woolf AS, Lopes FM, Ranjzad P, Roberts NA. Congenital disorders of the human urinary tract: recent insights from genetic and molecular studies. Front Pediatr. 2019;7:136.

4. Taghavi K, Sharpe C, Stringer MD. Fetal megacystis: a systematic review. JPediatr Urol. 2017;13(1):7-15.

5. Farrugia MK, et al. Experimental short-term fetal bladder outflow obstruction: I. Morphology and cell biology associated with urinary flow impairment. J Pediatr Urol. 2006;2(4):243-253.

6. Workman SJ, Kogan BA. Fetal bladder histology in posterior urethral valves and the prune belly syndrome. J Urol. 1990;144(2 pt 1):337-339.

7. Pipes GC, Creemers EE, Olson EN. The myocardin family of transcriptional coactivators: versatile regulators of cell growth, migration, and myogenesis. Genes Dev. 2006;20(12):1545-1556.

8. Wang D et al. Activation of cardiac gene expression by myocardin, a transcriptional cofactor for serum response factor. Cell. 2001;105(7):851-862.

9. Li S, Wang DZ, Wang Z, Richardson JA, Olson EN. The serum response factor coactivator myocardin is required for vascular smooth muscle development. Proc Natl Acad Sci U S A. 2003;100(16):9366-9370.

10. Huang J, et al. Myocardin is required for cardiomyocyte survival and maintenance of heart function. Proc Natl Acad Sci U S A. 2009;106(44):18734-18739.

11. Huang J, et al. Myocardin is required for maintenance of vascular and visceral smooth muscle homeostasis during postnatal development. Proc Natl Acad Sci U S A. 2015;112(14):4447-4452.

12. Wang Z, Wang DZ, Pipes GC, Olson EN. Myocardin is a master regulator of smooth muscle gene expression. Proc Natl Acad Sci U S A. 2003;100(12):7129-34.

13. Anderson CM, et al. Cooperative activation of cardiac transcription through myocardin bridging of paired MEF2 sites. Development. 2017;144(7):1235-1241.

14. Lek M, et al. Analysis of protein-coding genetic variation in 60,706 humans. Nature. 2016;536(7616):285-291.

15. Stuart HM, et al. Urinary tract effects of HPSE2 mutations. J Am Soc Nephrol. 2015;26(4):797-804.

16. Richer J, et al. R179H mutation in ACTA2 expanding the phenotype to include prune-belly sequence and skin manifestations. Am JMed Genet A. 2012;158A(3):664-668. 
17. Weber S, et al. Muscarinic acetylcholine receptor M3 mutation causes urinary bladder disease and a Prune-Belly-like syndrome. Am J Hum Genet. 2011;89(5):668-674.

18. Huang J, et al. Myocardin regulates expression of contractile genes in SM cells and is required for closure of the ductus arteriosus in mice. JClin Invest. 2008;118(2):515-525.

19. Singh S, et al. Identification of a unique transgenic mouse line that develops megabladder, obstructive uropathy, and renal dysfunction. JAm Soc Nephrol. 2007;18(2):461-471.

20. Singh S, et al. Transcriptional profiling of the megabladder mouse: a unique model of bladder dys- morphogenesis. Dev Dyn. 2008;237(1):170-186.

21. Ingraham SE, et al. Pathogenesis of renal injury in the megabladder mouse: a genetic model of congenital obstructive nephropathy. Pediatr Res. 2010;68(6):500-507.

22. Metcalfe PD, Meldrum KK. Sex differences and the role of sex steroids in renal injury. J Urol. 2006;176(1):15-21.

23. Seliger SL, Davis C, Stehman-Breen C. Gender and the progression of renal disease. Curr Opin Nephrol Hypertens. 2001;10(2):219-225.

24. Wang DZ, et al. Potentiation of serum response factor activity by a family of myocardin-related transcription factors. Proc Natl Acad Sci US A.
2002;99(23):14855-60.

25. Wilkie AO. The molecular basis of genetic dominance. J Med Genet. 1994;31(2):89-98.

26. McHugh KM. Megabladder mouse model of congenital obstructive nephropathy: genetic etiology and renal adaptation. Pediatr Nephrol. 2014;29(4):645-650.

27. Boghossian NS, et al. Rare copy number variants identified in prune belly syndrome. Eur JMed Genet. 2018;61(3):145-151.

28. Milewicz DM, et al. Genetic basis of thoracic aortic aneurysms and dissections: focus on smooth muscle cell contractile dysfunction. Annu Rev Genomics Hum Genet. 2008;9(1):283-302. 